\title{
réma
}

Tréma

$52 \mid 2019$

Peut-on penser le bonheur à l'école?

\section{L'activité empêchée, un stresseur environnemental de la pratique enseignante en classe de sciences?}

méthodologie de mesures des impacts des déplacements de l'enseignant dans la salle de science

Activity prevented, an environmental stressor of the teaching practice in science class? methodology for measuring the impacts of teacher shifts in the science room

Isabelle Lermigeaux-Sarrade et Laurent Jeannin

\section{(2) OpenEdition}

Journals

Édition électronique

URL : https://journals.openedition.org/trema/5566

DOI : $10.4000 /$ trema.5566

ISSN : 2107-0997

Éditeur

Faculté d'Éducation de l'université de Montpellier

Référence électronique

Isabelle Lermigeaux-Sarrade et Laurent Jeannin, «L'activité empêchée, un stresseur environnemental de la pratique enseignante en classe de sciences? », Tréma [En ligne], 52 | 2019, mis en ligne le 01 septembre 2019, consulté le 16 janvier 2023. URL : http://journals.openedition.org/trema/5566 ; DOI https://doi.org/10.4000/trema.5566

Ce document a été généré automatiquement le 16 janvier 2023.

Tous droits réservés 


\section{L'activité empêchée, un stresseur environnemental de la pratique enseignante en classe de sciences?}

méthodologie de mesures des impacts des déplacements de l'enseignant dans la salle de science

Activity prevented, an environmental stressor of the teaching practice in science class? methodology for measuring the impacts of teacher shifts in the science

room

Isabelle Lermigeaux-Sarrade et Laurent Jeannin

\section{Introduction}

1 Le constat que les enseignants nous livrent sur leurs conditions d'exercice de leur métier nous indique qu'il n'est pas toujours facile d'être bien dans ses mouvements et dans ses déplacements en classe, dans le but d'interagir avec les élèves :

...pour moi la disposition de la salle n'est pas pratique du tout parce que là du coup on voit les tables... que ça ... non c'est vrai ça c'est ... franchement pénible

...en plus là c'est encore pas une classe qui bouge ils sont sympas c'est vrai que sur une classe où il faut souvent se déplacer la surtout avec les troisièmes la t'es vraiment obligé de circuler parce que sinon ils font des bêtises je trouve que c'est vraiment pas...

Plusieurs questions de recherche peuvent se construire à partir de ce constat : dans une perspective d'habiter l'espace classe, comment les enseignants prennent-ils en charge leurs contraintes spatiales? Est-ce un frein ou un levier d'innovation dans leurs pratiques? Ou encore quelles sont les conditions pour exercer convenablement le métier d'enseignant et gérer au sens de M. Altet (1994) la dynamique d'interactions nécessaire à la construction de sens ?... 
3 Fischer (2011) démontre que l'espace construit, i.e. celui qui est perçu et exploité par un collectif et plusieurs individualités n'est pas neutre. Il conditionne en partie nos relations avec les autres. L'être humain maintient un espace entre lui et les autres et construit cet espace autour de lui, à la maison et dans son environnement professionnel. L'espace est une «dimension cachée » (Hall, 1966), dont l'individu se sert de façon souvent inconsciente. Il est un espace à la fois de protection et de construction. Les interactions dans la classe ont lieu dans un espace dont l'organisation peut stimuler ou inhiber les relations entre les élèves (Weinstein, 1992), comme par exemple le tableau ou la table des élèves qui va conditionner des types d'interactions.

Cet article présente une méthodologie pour explorer des configurations de classe en situation réelle permettant à l'enseignant d'être en proximité d'élèves quand ils échangent et d'étudier ce qui peut empêcher l'enseignant dans ses déplacements et donc son impact sur sa pratique en classe (Robert 1999, Rogalski, 1999). Pour ce faire, nous reprenons la notion de stresseur environnemental (Selye, 1946) afin de déterminer ce qui empêche physiquement et/ou conditionne une part de l'activité enseignante en classe et qui peut être facteur de mal-être au travail. Nous appliquons notre méthode à quatre espaces de classe de sciences en collège. A partir de ces données, nous travaillons à l'analyse des configurations spatiales d'espaces propices aux interactions de classe, des espaces «scolairement sociopètes» (Hall, 1966), susceptibles de favoriser les interactions, facteur de bien-être des enseignants au travail et de renforcer la qualité des processus d'apprentissages au quotidien.

\section{Facteurs de stress et environnement de travail de l'enseignant}

5 Rascle et Bergugnat, (2016) suggèrent une interdépendance entre la qualité de vie des enseignants au travail et celle des élèves. L'approche ergonomique du travail (Karwowski, 2005) envisage les interactions entre les individus et leur environnement de travail comme un facteur favorisant une meilleure qualité de vie dans sa pratique professionnelle, en améliorant la sécurité, la performance, la fiabilité, l'environnement de travail, la facilité d'utilisation, et en réduisant les erreurs, la fatigue, le stress physique, les pertes de temps et de matériel (Chapanis, 1996, cité par Karwowski, 2005). Nous nous intéressons dans ce qui suit aux conditions de l'agencement de l'espace classe pouvant être des stresseurs environnementaux au sens de H. Seyle.

\section{Enseigner, une histoire de compromis}

6 La tâche de l'enseignant est complexe et multiforme (Bucheton \& Soulé, 2009), le plaçant dans une tension permanente entre les objectifs qu'il cherche à atteindre et ses conditions effectives d'enseignement (Ria, 2008). L'activité de l'enseignant résulte d'un compromis (Guérin, Laville, Daniellou, Duraffourg, \& Kerguelen, 1991), d'un arbitrage entre de multiples prescriptions et un contexte caractérisé par l'environnement physique, les moyens technologiques et les interactions entre les individus (Leplat, 1997). 


\section{Pratiquer avec des injonctions contradictoires et des comportements perturbateurs}

7 La méta-analyse de Stewart (2015) mentionne différents facteurs de stress concernant le métier d'enseignant, en particulier les effectifs de classe élevés, les injonctions contradictoires, et les comportements perturbateurs des élèves au quotidien. Ce dernier élément pourrait être le facteur le plus pesant dans le stress de l'enseignant.

Laugaa (2004) a observé que les enseignants néo-titulaires en difficulté professionnelle, en situation de stress voire d'épuisement professionnel, empruntent fréquemment une stratégie d'adaptation consistant à sélectionner un style d'enseignement traditionnel, directif et contraignant pour les élèves, pour gérer les comportements dans la classe. Il est à noter que la configuration frontale des salles, héritée des lois Ferry, a été pensée initialement pour le cours magistral qui s'est généralisé à cette époque (Le Coeur, 2011), et correspond à ce style d'enseignement et à ce besoin de contrôle visuel et aussi physique. Cette configuration des salles qui se retrouve aussi pour les salles de sciences, véhicule une représentation et un message contradictoire avec l'enseignement des sciences fondé sur l'investigation, introduit en France dès 2005. Ce type d'approche suppose une part accrue des travaux en petits groupes encourageant le débat scientifique et des phases collectives d'institutionnalisation. Le décret du 31 mars 2015 définissant le socle commun de connaissances, de compétences et de culture officialise cette place au travail de groupe au collège.

\section{Comment agir, si nos actions sont empêchées?}

Nous faisons l'hypothèse que la disposition fixe des paillasses en science est une contrainte que l'enseignant doit gérer, même si la configuration ne répond pas à ses besoins d'organisation de l'espace. Les actions empêchées peuvent faire perdre du sens à l'activité et être responsables de souffrance et de stress au travail en amputant le pouvoir d'agir de l'individu (Clot, 2014, 2015). Pour Grangeat (2010), l'enseignant a un répertoire d'action, qui lui permet d'agir dans la classe en fonction du but qu'il s'est fixé. Il repère des indices pertinents au fil de la situation de classe, et agit en fonction de connaissances professionnelles issues de son parcours et de son expérience professionnelle. Les déplacements dans la classe sont des micro-actions du quotidien. Elles peuvent être considérées comme la conséquence de multiples micro-décisions prises par l'enseignant au fil de l'activité (Lermigeaux-Sarrade, 2018). Nous faisons l'hypothèse qu'être gêné dans ses déplacements pourrait constituer une limitation du pouvoir d'agir de l'enseignant et par conséquent un facteur de stress au travail.

\section{Configuration de l'espace et pratiques enseignantes}

\section{Proxémie et interactions dans la classe}

10 «La proxémie définit l'ensemble des observations et des théories concernant l'usage de l'espace par l'homme. » (Hall, 1966 ; p. 129). L'enseignant se déplace dans la classe, s'approchant d'un groupe, s'éloignant d'un autre, en oscillant dans plusieurs espaces proxémiques, en passant de la sphère publique à la sphère sociale, en entrant dans les sphères personnelles des élèves. Pour Forest (2006), l'enseignant utilise la proxémie dans les 
interactions didactiques en se déplaçant selon une gestuelle complexe et en réservant des lieux spécifiques pour une typologie d'interactions: l'énonciation, le questionnement à la classe, l'institutionnalisation... Forest (ibid) identifie et cartographie des lieux que l'enseignant utilise préférentiellement quand il s'adresse à la classe entière. Cette utilisation stratégique de l'espace est également repérée par Moulin (2004), qui considère que les déplacements et placements permettent à l'enseignant de contrôler les comportements et de mobiliser l'attention dans une communication non-verbale partagée avec les élèves. Il identifie également des emplacements privilégiés utilisés par l'enseignant lors de rituels comme l'accueil en classe.

11 Les enseignants novices s'interrogent dès la prérentrée sur leurs placements et déplacements dans la classe. En réponse à ces inquiétudes, quelques dispositifs de formation spécifiques concernant la posture, le corps et la voix sont mis en place en formation initiale des enseignants, mais leur place reste très limitée (Jourdan, 2014). Au regard des contenus des maquettes de formation portant sur cet enseignement, il est d'avantage question du placement du corps et de la voix dans une perspective d'autorité et de présence dans l'espace classe, que d'apprendre à se situer dans un ou plusieurs espaces différenciant au regard des pratiques.

\section{Mobilité et facilité d'accès}

12 Le triplet de mobilité de la salle de classe (Jeannin, 2017) vise à rendre compte de la capacité inerte de l'espace classe, du point de vue des possibilités de déplacements relatifs et absolus. Il est basé sur trois métriques, à savoir: alpha pour l'espace pris quand les élèves sont assis, beta qui rend compte de l'espace d'évolution possible pour l'enseignant et gamma qui correspond au coefficient de mobilité pour l'enseignant et pour les élèves. L'étude de 1800 configurations de salles montre que le triplet de mobilité le plus favorable dans un espace contraint est une organisation spatiale en îlot. Le travail proposé ici vient ré-interroger ce résultat en y incluant la pratique enseignante dans une perspective double, à savoir l'espace qui contraint un déplacement et l'espace qui est facteur de régulation.

\section{Espace social}

13 La configuration frontale de la salle de classe définit un espace social, réseau de relations et support de la communication et des interactions (Altet, 1994 ; Fischer \& Fousse, 2002). On peut se demander si dans une autre configuration, on retrouverait la stratégie de recours à un style d'enseignement traditionnel identifiée par Laugaa (2004).

Il arrive que l'enseignant utilise le placement des élèves dans la classe pour contrôler leurs comportements (Rißler, Bossen, \& Blasse, 2014). Ce placement a une incidence sur leurs performances (Brooks, 2011, 2012; Perkins et Wieman, 2005) et sur leurs comportements (Weinstein, 1992). L'engagement des élèves dans la tâche est différent selon leur disposition et selon que la tâche est individuelle ou collaborative (Wannarka \& Ruhl, 2008; Weinstein, 1992). Pour renforcer la qualité de la situation d'apprentissage, il faudrait que la disposition choisie soit cohérente avec la communication souhaitée entre les élèves. Par exemple, une disposition en îlots incite 
les élèves au travail collaboratif mais s'avère contre-productive pour un travail individuel. Weinstein (ibid) suggère d'intégrer une "environmental competence » dans la formation initiale et continue des enseignants.

Ces différents constats rejoignent les conclusions d'études plus anciennes concernant les effets de la disposition des individus autour d'une table sur la qualité de la communication et la fréquence des interactions. La qualité du contact visuel entre les individus en interaction ressort de ces différentes études comme un élément déterminant de la qualité de structure de communication (Howells \& Becker, 1962; Lécuyer, 1975 ; Sommer, 1961, 1965, 1967).

La communication entre les élèves semble également être affectée par la facilité d'accès de l'enseignant à leur espace de travail (Issaadi \& Jaillet, 2017).

\section{Contraintes de passabilité attachées à la configuration}

La passabilité entre deux obstacles est une caractéristique qui ressort d'études concernant l'action guidée par la perception visuelle des distances et impliquant des obstacles physiques ou des personnes (Efran \& Cheynes, 1973 ; Warren \& Wang, 1987, cités par Morgado, 2014). D'une part, ces études expérimentales montrent qu'il existe un seuil critique entre deux obstacles physiques dépendant de la largeur d'épaule du sujet et de la largeur de l'ouverture. D'autre part, elles indiquent que la perception proxémique de la sphère personnelle intervient dans la décision de passer ou non entre des personnes.

Pour l'enseignant, la décision de passer entre deux rangs pour interagir avec un groupe d'élèves est donc soumise à des contraintes qui ne sont pas purement géométriques, mais qui dépendent aussi de ses caractéristiques personnelles, morphologiques, et culturelles, et de celles des élèves.

\section{Problématique}

19 Poser la question de la mobilité possible comme facteur de mieux-être dans la relation pédagogique interroge plusieurs champs. Le premier est celui qui est directement lié à ce qui est possible de faire avec la configuration de l'espace classe, donc hors obstacles pérennes, comme des tables fixées au sol, ou encore ponctuels liés aux personnes présentes et à la pédagogie mise en place. Le second vise à interroger les conditions de régulation des déplacements de l'enseignant : directement liées à l'espace, à son style d'enseignement ou encore aux indices pris en situations qui l'engagent dans des nouvelles actions. Nous déclinons cette problématique en trois questions de recherche, à savoir :

1. Quelles configurations permettent à l'enseignant d'être plus proche de ses élèves dans les interactions avec eux?

Nous faisons l'hypothèse qu'une configuration qui facilite la mobilité comme celle en îlots (Jeannin, 2017) améliore la proximité réelle de l'enseignant, donc est un élément facilitateur de la relation pédagogique et donc tend à réduire le stress et aura pour conséquence un mieux-être dans son geste professionnel.

2. Quelles contraintes d'accessibilité modifient la proximité réelle de l'enseignant? Nous faisons l'hypothèse que des configurations qui présentent des obstacles devant être contournés altèrent la proximité réelle de l'enseignant. 
3. Quelles contraintes de passabilité modifient les déplacements et les placements de l'enseignant dans ses interactions avec les élèves?

Nous faisons ici l'hypothèse que des configurations qui présentent un espace entre rangs trop faible modifient les placements de l'enseignant.

\section{Méthode de mesure}

20 L'objectif est 1) initier un protocole de mesure de la facilité d'accès théorique, 2) le tester en situation naturelle et 3) identifier à l'aide d'un encodage croisé quelles seraient des configurations qui facilitent les déplacements et les placements, qui rendent fluides et qui améliorent les interactions.

\section{Protocole de mesure de l'accessibilité potentielle théorique}

21 L'objectif est ici de se doter d'une détermination théorique de la facilité d'accès dans un espace de classe. Cette notion de facilité d'accès est très utilisée et depuis longtemps, en géographie et en urbanisme où elle complète la notion de distance (Dumolard, 1999). Pour les géographes, l'accessibilité est une mesure concrète, qui peut se mesurer de différentes façons selon la situation considérée. Sa définition prend en compte la position d'un nœud sur un réseau et les différents chemins minimaux qui permettent d'accéder à ce nœud depuis les autres nœuds du réseau (Dumolard, 1999 ; Vickerman, 1974).

22 Il s'agit ici de contextualiser cette définition dans la salle de classe, en considérant que l'accessibilité est une réalité qui dépend de la nature de l'activité qui se déroule. Si on se place dans le cas d'un travail en groupe, chaque groupe d'élèves peut être ainsi considéré comme un nœud auquel l'enseignant peut accéder, depuis chacun des autres groupes d'élèves, disposés dans la classe comme les nœuds d'un réseau. Dans le cas d'une intervention de l'enseignant s'adressant à toute la classe, le tableau peut être considéré comme le nœud auquel l'enseignant va accéder, depuis le lieu précédent d'interaction. Vickerman (1974) propose un calcul d'accessibilité relative à un nœud du réseau, et un calcul d'accessibilité globale pour l'ensemble des nœuds d'un réseau.

Lermigeaux-Sarrade (2018) a adapté ces calculs dans la salle de classe, dans le cas du travail en groupe. L'accessibilité potentielle vis-à-vis d'un groupe représente pour elle la distance moyenne topologique franchie par l'enseignant pour se placer devant un groupe, depuis n'importe lequel des autres groupes d'élèves présents dans la salle lors de la séance. Il s'agit d'une distance topologique, car l'enseignant doit contourner les obstacles dans la classe ce qui ne peut pas être intégré dans une mesure « classique » de distance. C'est aussi une distance théorique, qui correspond au comportement mécanique d'un « enseignant-robot » qui partagerait le temps de la séance également entre les groupes et suivrait un chemin minimum d'un groupe à l'autre. Il est à noter que ce calcul des distances d'accessibilité potentielle dans la classe tient compte d'éléments fixes et semi-fixes. Les éléments fixes sont les murs, la géométrie de la classe. Les éléments semi-fixes sont les nœuds du réseau, que Lermigeaux-Sarrade détermine à partir des points optimaux d'interaction (PIO) de l'enseignant avec les groupes d'élèves présents lors de la séance. 
La figure 1 montre comment est établi le réseau ainsi défini pour une salle de sciences dont la configuration spécifique est désignée dans ce qui suit par «configuration hybride ", à savoir avec une organisation mixte de la salle de classe (figure 5).

Figure.1 : Plan de classe (à gauche) et réseau des Points d'Interaction Optimaux (à droite) - salle de classe en configuration Hybride.

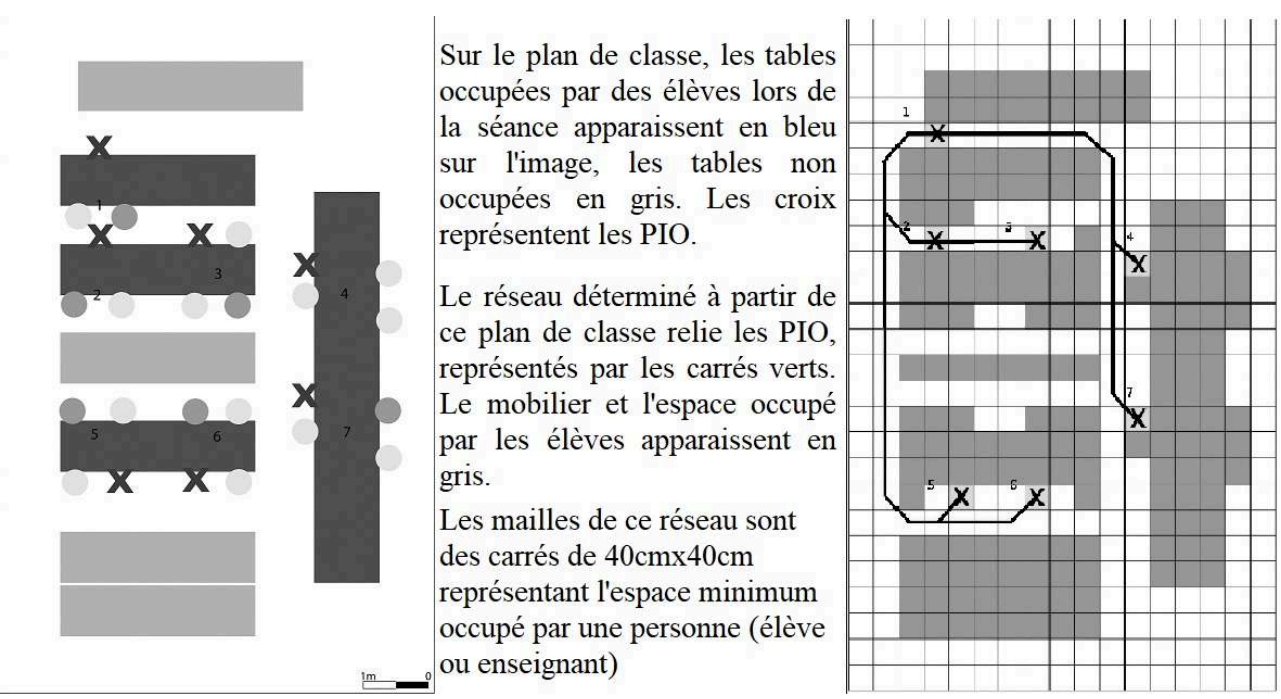

Pour déterminer l'accessibilité vis-à-vis d'un groupe, la première étape suivie par Lermigeaux-Sarrade consiste à représenter la salle et son mobilier, de manière précise. La deuxième étape est de repérer le placement des élèves. La troisième étape est le choix des Points d'Interactions Optimaux (PIO) de l'enseignant avec chaque groupe d'élève. Ces points sont choisis de manière à optimiser le contact visuel avec les élèves du groupe. Ils figurent sur le plan de classe (figure 1) sous la forme de croix.

En reprenant la définition d'accessibilité de Vickerman (1974), il est à noter que les distances d'accessibilité sont d'autant plus grandes que l'accès spécifique à chaque groupe ou que l'accès global à l'espace de classe est difficile. En effet, la valeur d'accessibilité dans une salle où chaque déplacement d'un groupe à l'autre est long est plus grande que celle qu'on obtiendra dans une salle où l'enseignant rayonne entre les groupes à partir de trajets courts.

\section{Mesure des déplacements et de la proximité réelle en situation de classe}

Un dispositif de capture des déplacements (Lermigeaux-Sarrade, 2018) a été mis au point pour enregistrer à chaque seconde la position de l'enseignant dans la classe. L'enseignant porte un gilet émettant des spots infra-rouges intermittents, les informations reçues par deux caméras placées dans deux angles de la salle permettent de connaître sa position. 
Figure.2 : A gauche, image d'une des caméras infra-rouge et à droite le principe de repérage.
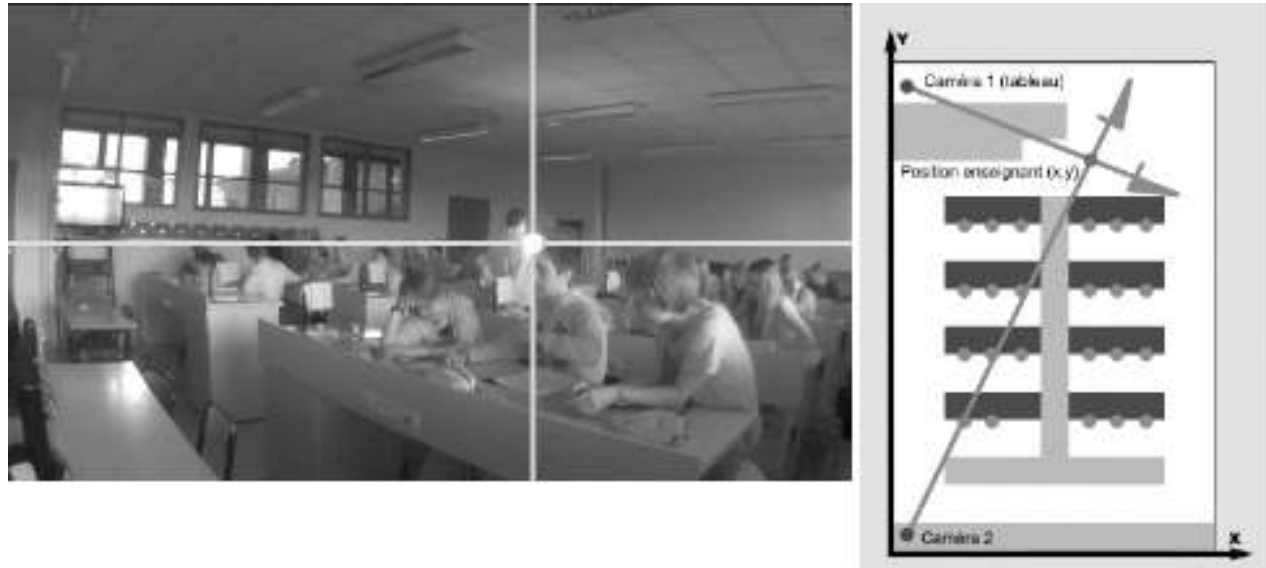

Ces données spatiales sont visualisées d'une part sous la forme de cartographies point par point (figure 3) et d'autre part sous la forme de cartes de densité (figure 4), ces deux modes de visualisation complémentaires permettant une analyse qualitative des placements et déplacements.

Figure. 3 : Cartographie points par points / Figure.4 : Densité de présence de l'enseignant

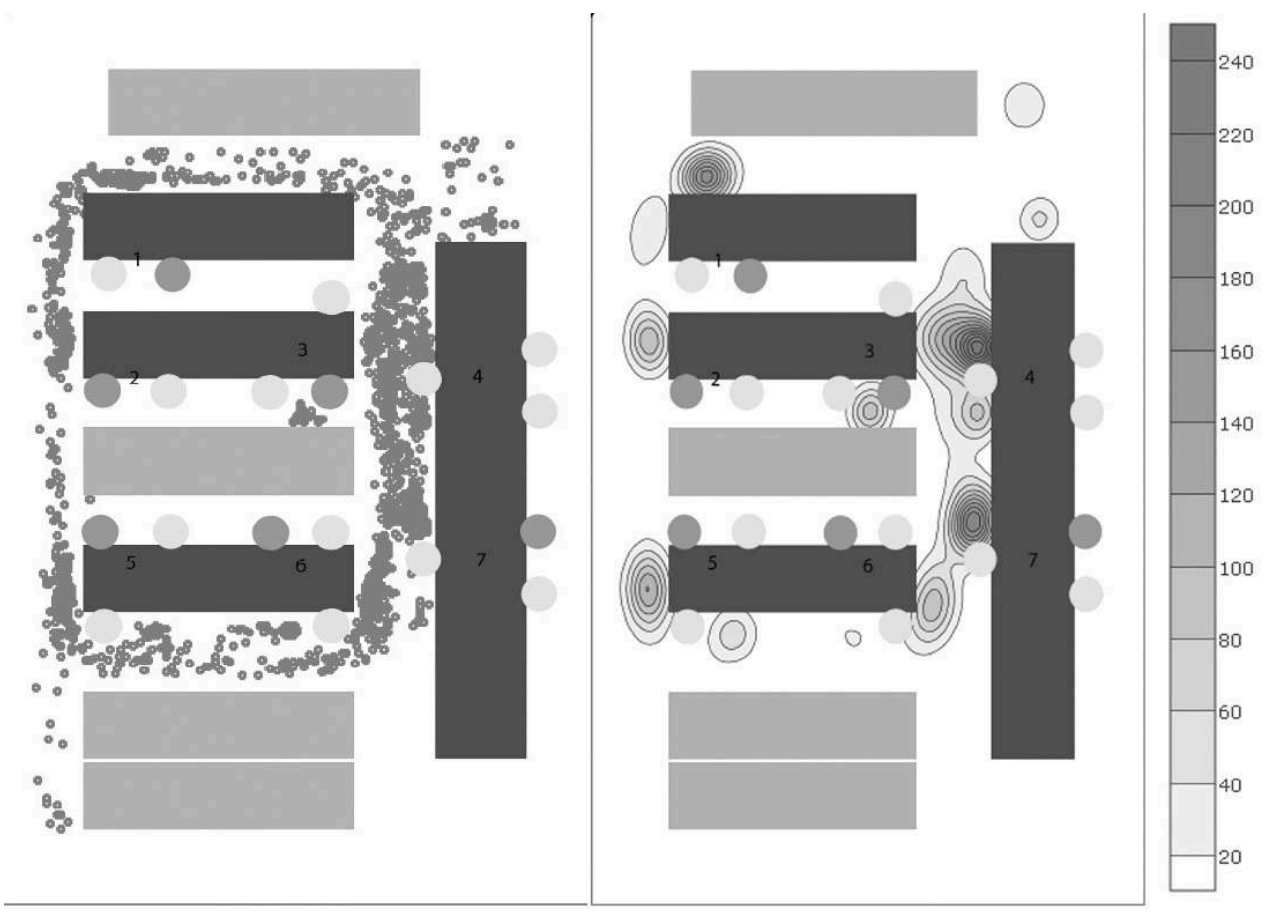

Pour une même séance, la cartographie point par point dessine le parcours de l'enseignant, sans pour autant rendre compte de l'aspect temporel des déplacements. La densité de présence la complète en montrant où l'enseignant stationne. Plus le temps d'arrêt est long, plus la couleur des zones est foncée, les zones blanches étant des zones où l'enseignant passe sans s'arrêter. 


\section{Comparaison de configurations} dans quatre configurations de classe, îlots, bus, hybride et peigne, représentées sur la figure 5. Nous examinons dans ce qui suit les résultats concernant ces quatre espaces de classe.

Figure 5 : Configurations îlots, bus, hybride, peigne
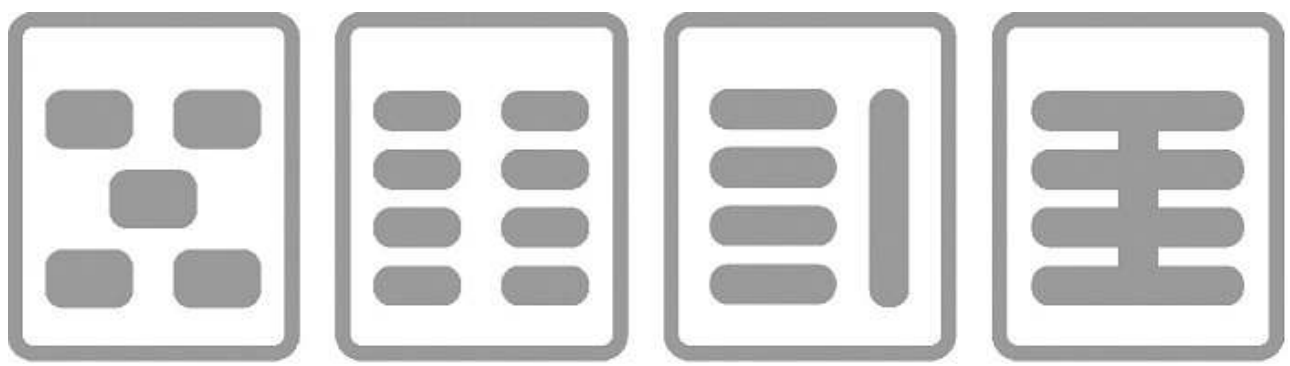

La configuration îlots comporte cinq tables avec cinq places possibles par table dont une table centrale. Elle a été retenue car elle est de plus en plus utilisée dans les établissements (Jeannin, 2017) et parce qu'elle répond aux besoins d'un enseignement des sciences fondés sur l'investigation inscrit dans les prescriptions actuelles en matière d'enseignement des sciences (cf note $n^{\circ} 1$ de cet article pour les références scientifiques)

La configuration bus est une disposition classique en rangs de deux tables de trois places avec une travée centrale.

La configuration hybride comporte des rangs de tables de quatre places et une longue paillasse latérale.

La configuration peigne est formée de paillasses de trois places possibles attachées à une longue paillasse centrale comme les dents d'un double peigne. Elle ne comporte pas de travée de circulation centrale. Par l'absence de cet espace de circulation, cette configuration nous paraît présenter la plus mauvaise accessibilité.

Le tableau 1 présente les résultats des calculs d'accessibilité potentielle de ces quatre configurations.

Tableau 1. Accessibilité potentielle - Configurations Bus, Peigne, Hybride et llots.

\begin{tabular}{|l|l|l|l|l|l|l|l|l|l|l|l|l|l|}
\hline & & $n$ & $M$ & $95 \% C I$ & $S D$ & & & $n$ & $M$ & $95 \% C I$ & $S D$ & $t$ & $p$ \\
\hline A & Bus1 & 8 & 4,2 & {$[3,5 ; 4,8]$} & 0,7 & B & Peigne1 & 8 & 7,2 & {$[5,2 ; 9,1]$} & 2,3 & & \\
\hline B & Bus2 & 8 & 4,6 & {$[4,0 ; 5,2]$} & 0,7 & A & Peigne2 & 7 & 8 & {$[5,8 ; 10,3]$} & 2,4 & & \\
\hline & Bus12 & $\mathbf{1 6}$ & $\mathbf{4 , 4}$ & {$[\mathbf{4 , 0 ; 4 , 8}]$} & $\mathbf{0 , 7}$ & & Peigne & $\mathbf{1 5}$ & $\mathbf{7 , 6}$ & {$[\mathbf{6 , 3 ; 8 , 8}]$} & $\mathbf{2 , 3}$ & $\mathbf{- 5 , \mathbf { 0 8 }}$ & $\mathbf{0 , 0 0 0 1}$ \\
\hline D & Bus3 & $\mathbf{8}$ & 4,3 & {$[3,3 ; 5,3]$} & 1,2 & C & Hybride1 & 7 & 5,2 & {$[3,2 ; 7,1]$} & 2,1 & & \\
\hline C & Bus4 & 7 & 3,7 & {$[2,6 ; 4,8]$} & 1,1 & D & Hybride2 & 7 & 5,1 & {$[3,6 ; 6,6]$} & 1,6 & & \\
\hline & Bus34 & $\mathbf{1 5}$ & $\mathbf{4 , 0}$ & {$[\mathbf{3 , 4 ; 4 , 7}]$} & $\mathbf{1 , 2}$ & & Hybride & $\mathbf{1 4}$ & $\mathbf{5 , 1}$ & {$[\mathbf{4 , 1 ; 6 , 2 ]}$} & $\mathbf{1 , 8}$ & $\mathbf{- 1 , 9 4}$ & $\mathbf{0 , 0 6}$ \\
\hline
\end{tabular}




\begin{tabular}{|l|l|l|l|l|l|l|l|l|l|l|l|l|l|}
\hline F & Bus5 & 5 & 4,1 & {$[3,3 ; 4,9]$} & 0,6 & E & Ilots1 & 5 & 3,2 & {$[2,1 ; 4,1]$} & 0,8 & & \\
\hline E & Bus6 & 5 & 4,3 & {$[3,2 ; 5,4]$} & 0,9 & F & Ilots2 & 5 & 3,1 & {$[2,3 ; 3,8]$} & 0,6 & & \\
\hline & Bus56 & $\mathbf{1 0}$ & $\mathbf{4 , 0}$ & {$[\mathbf{3 , 4 ; 4 , 7}]$} & $\mathbf{1 , 2}$ & & Ilots & $\mathbf{1 0}$ & $\mathbf{3 , 1}$ & {$[\mathbf{2 , 6 ; 3 , 6}]$} & $\mathbf{0 , 7}$ & $\mathbf{3 , 5 4}$ & $\mathbf{0 , 0 0 2}$ \\
\hline & Bus & $\mathbf{4 1}$ & $\mathbf{4 , 2}$ & {$[\mathbf{3 , 9 ; 4 , 5}]$} & $\mathbf{0 , 9}$ & & & & & & & & \\
\hline
\end{tabular}

Le premier constat est que les distances d'accessibilité potentielle de ces quatre configurations apparaissent comme différentes (Tableau 1). Elles s'échelonnent de 3,1m $($ IC95 \% $=[2,6 ; 3,6])$ pour la configuration îlots à 7,6m $($ IC95\% $\%[6.3 ; 8.8])$ pour la configuration peigne. Les calculs montrent que la distance moyenne théorique parcourue par l'enseignant entre deux groupes est presque deux fois plus longue en configuration Peigne que dans la configuration Bus.

\section{Résultats}

\section{Résultat 1 : Mobilité et proximité}

Notre première question de recherche examine quelles configurations permettent à l'enseignant d'être plus proche d'élèves avec lesquels il interagit. L'hypothèse envisagée est qu'une configuration qui facilite la mobilité comme la configuration en îlots (Jeannin, 2017) améliore la proximité réelle de l'enseignant.

Les résultats montrent des différences dans l'activité des trois enseignants, selon la configuration, qui apparaissent dans les mesures des proximités réelles vis-à-vis des groupes d'élèves (Tableau 2), obtenues à partir des déplacements effectifs. Ces valeur de proximité réelle s'échelonnent de $2,9 \mathrm{~m}(\mathrm{IC} 95 \%=[2,3 ; 3,5])$ pour la configuration îlots à $6,1 \mathrm{~m}($ IC95 $\%=[5,3 ; 7,0])$ pour la configuration peigne.

Tableau 2. Proximité réelle - Configurations Bus, Peigne, Hybride et llots.

\begin{tabular}{|c|c|c|c|c|c|c|c|c|c|c|c|c|c|c|}
\hline \multicolumn{2}{|c|}{ Enseignant } & & $n$ & M & $95 \% \mathrm{CI}$ & $S D$ & & & $n$ & $M$ & $95 \% C I$ & $S D$ & $t$ & $p$ \\
\hline & A & Bus1 & 8 & 3,4 & {$[2,8 ; 4,0]$} & 0,7 & B & Peigne1 & 8 & 5,7 & {$[4,3 ; 7,1]$} & 1,6 & & \\
\hline & B & Bus2 & 8 & 3,4 & {$[3,1 ; 3,8]$} & 0,4 & A & Peigne2 & 7 & 6,6 & {$[5,6 ; 7,7]$} & 1,2 & & \\
\hline T1 & & Bus12 & 16 & 3,4 & {$[3,1 ; 3,7]$} & 0,6 & & Peigne & 15 & 6,1 & {$[5,3 ; 7,0]$} & 1,5 & $-6,71$ & $<0,0001$ \\
\hline & D & Bus3 & 8 & 4,1 & {$[3,6 ; 4,7]$} & 0,7 & C & Hybride 1 & 7 & 4,6 & {$[4,0 ; 5,3]$} & 0,7 & & \\
\hline & C & Bus4 & 7 & 3,3 & {$[2,5 ; 4,1]$} & 0,9 & D & Hybride2 & 7 & 4,4 & {$[3,0 ; 5,7]$} & 1,4 & & \\
\hline T2 & & Bus34 & 15 & 3,7 & {$[3,3 ; 4,2]$} & 0,9 & & Hybride & 14 & 4,5 & {$[3,9 ; 5,1]$} & 1,1 & $-2,06$ & 0,05 \\
\hline & $\mathrm{F}$ & Bus5 & 5 & 3,3 & {$[2,3 ; 4,2]$} & 0,8 & $\mathrm{E}$ & Ilots1 & 5 & 2,5 & {$[1,3 ; 3,7]$} & 1,0 & & \\
\hline & E & Bus6 & 5 & 3,6 & {$[3,3 ; 4,0]$} & 0,3 & $\mathrm{~F}$ & Ilots2 & 5 & 3,2 & {$[2,5 ; 4,0]$} & 0,6 & & \\
\hline
\end{tabular}




\begin{tabular}{|l|l|l|l|l|l|l|l|l|l|l|l|l|l|l|}
\hline T3 & & Bus56 & 10 & 3,4 & {$[3,0 ; 3,9]$} & 0,6 & & Ilots & 10 & 2,9 & {$[2,3 ; 3,5]$} & 0,9 & 1,77 & 0,09 \\
\hline
\end{tabular}

Pour les trois enseignants et les 82 mesures concernant les groupes d'élèves, la proximité réelle apparaît comme corrélée à l'accessibilité potentielle ( $n=80, t=6.2$, $p<0.0001, r=0.545$ ).

Figure. 6 : Accessibilité potentielle et proximité réelle - synthèse des résultats

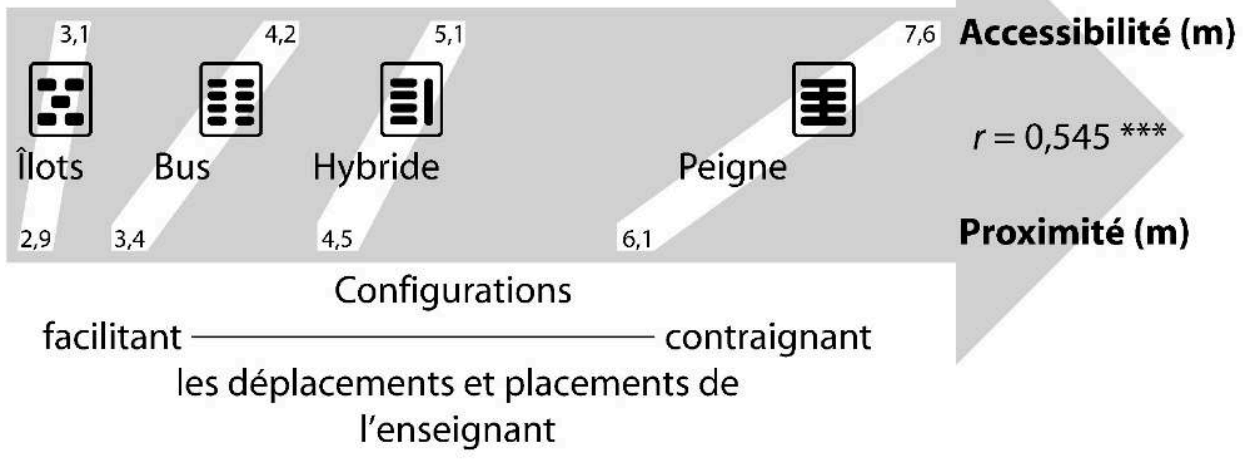

Les déplacements effectifs de l'enseignant semblent donc être en partie déterminés par l'accessibilité potentielle de la configuration.

La configuration en îlots, dans laquelle la mobilité est la plus grande (Jeannin, 2017), a la plus petite valeur d'accessibilité potentielle, et la plus petite valeur de proximité réelle. Elle permet donc à l'enseignant d'être plus proche des élèves dans ses interactions ce qui va dans le sens de notre première hypothèse et des résultats de Jeannin (2017) avec le triplet de mobilité.

\section{Résultat 2 : Accessibilité potentielle et proximité réelle}

Notre deuxième question de recherche porte sur comment les contraintes d'accessibilité modifient la proximité réelle de l'enseignant. L'hypothèse était que des configurations de forme complexe présentant des obstacles devant être contournés altèrent la proximité réelle de l'enseignant.

La configuration en peigne répond à ces critères, l'absence de travée centrale constitue un obstacle et impose des contournements. Il apparaît qu'elle a la plus grande valeur d'accessibilité potentielle, par conséquent elle présente la plus mauvaise accessibilité. Elle a aussi la plus grande valeur de proximité réelle. C'est la configuration dans laquelle l'enseignant est le moins proche des élèves dans ses interactions. L'obstacle que constitue l'absence de travée centrale et la complexité de la configuration apparaissent donc bien comme associés à une altération de la proximité réelle, ce qui va dans le sens de notre deuxième hypothèse. 


\section{Résultat 3 : placements et contraintes de passabilité}

Notre troisième question recherche les contraintes de passabilité modifiant les placements de l'enseignant dans ses interactions avec les élèves. L'hypothèse était que des configurations qui présentent un espace entre rangs trop faible modifient les placements de l'enseignant

Pour répondre à cette question, nous examinons plus particulièrement les résultats concernant les déplacements d'une enseignante de Sciences et Vie de la Terre (SVT) dans la configuration hybride (Lermigeaux-Sarrade, 2018).

L'examen de la cartographie des déplacements points par points (figure 7) fait apparaître "en creux» des déplacements et des placements empêchés, que nous analysons ici à partir de contraintes de passabilité.

Sur les cartographies, le tableau et le bureau de l'enseignante sont en haut de l'image. Les deux tables en fond de classe sont occupées par du matériel et des ordinateurs. Lors de la séance $1, l^{\prime}$ enseignante a placé les élèves en utilisant trois paillasses contiguës au centre de la salle. Lors de la séance 2, elle laissé une paillasse libre qui espace un peu plus les groupes d'élèves.

Pour les deux séances, l'observation montre le même type de parcours, formant un anneau. L'enseignante ne s'engage pas ou peu entre les rangs. L'observation vidéo montre que lorsqu'elle passe entre les rangs, c'est pour répondre à une demande spécifique des élèves.

Figure.7 : Cartographies point par point - configuration Hybride - à gauche, séance 1 - à droite, séance 2.
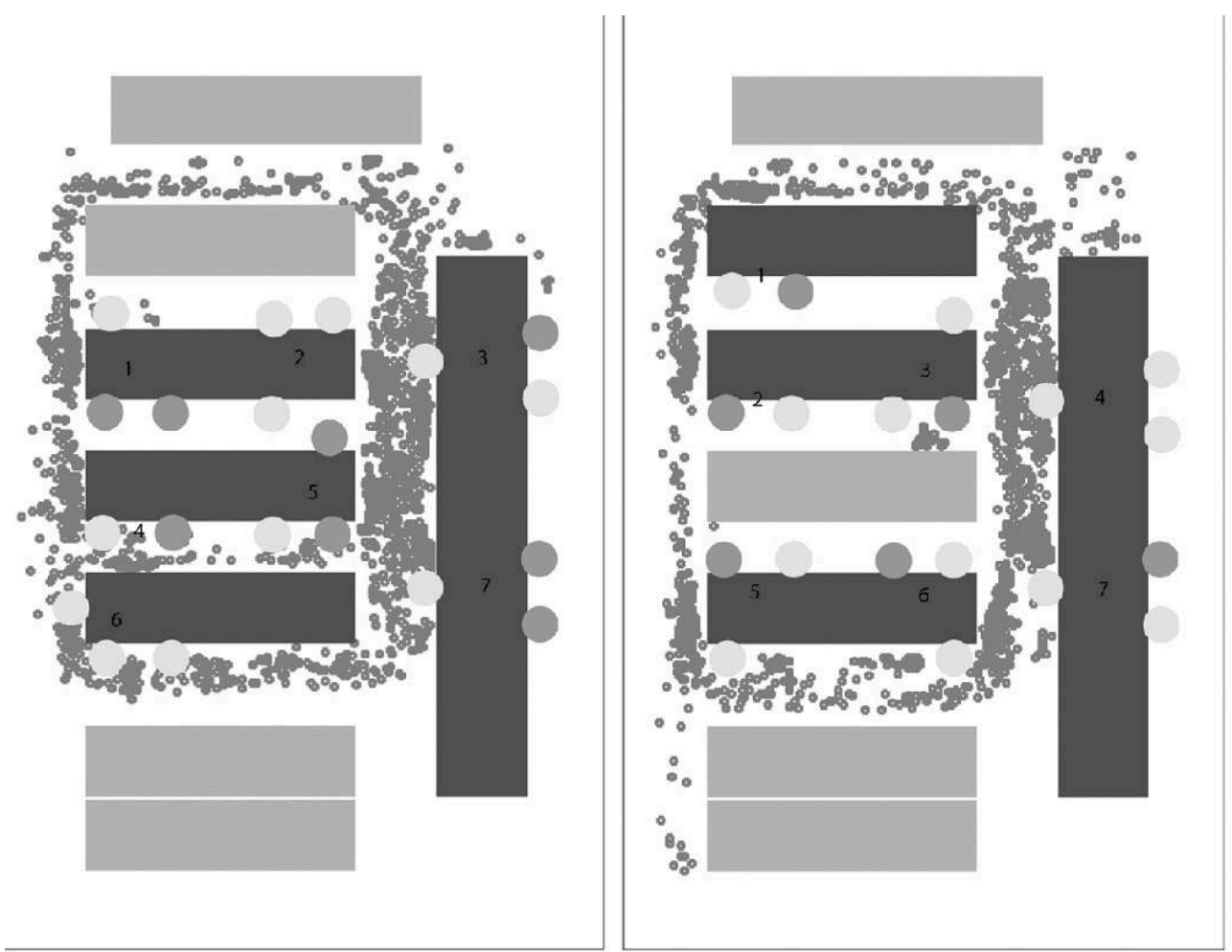

Les mesures montrent que la largeur du passage dans l'anneau des déplacements est de $90 \mathrm{~cm}$ (Tableau 3). La distance entre rangs de $60 \mathrm{~cm}$ est une contrainte qui modifie 
effectivement les déplacements de l'enseignante pour l'interaction avec les groupes, ce qui va dans le sens de notre hypothèse.

Tableau 3. Dimension des travées dans la configuration Hybride

\begin{tabular}{|l|l|}
\hline Largeur des tables $(\mathrm{cm})$ & 80 \\
\hline Espace entre les rangs $(\mathrm{cm})$ & 60 \\
\hline Largeur travée centrale $(\mathrm{cm})$ & 90 \\
\hline Largeur travée latérale gauche $(\mathrm{cm})$ & 98 \\
\hline Largeur travée latérale droite $(\mathrm{cm})$ & 90 \\
\hline
\end{tabular}

L'enseignante est plus souvent en position latérale que face à face (figure 8). La difficulté à circuler entre les rangs semble l'empêcher de se placer, ou la contraint à cette position.

Figure.8 : Densités de présence - configuration Hybride - à gauche, séance 1 - à droite, séance 2.
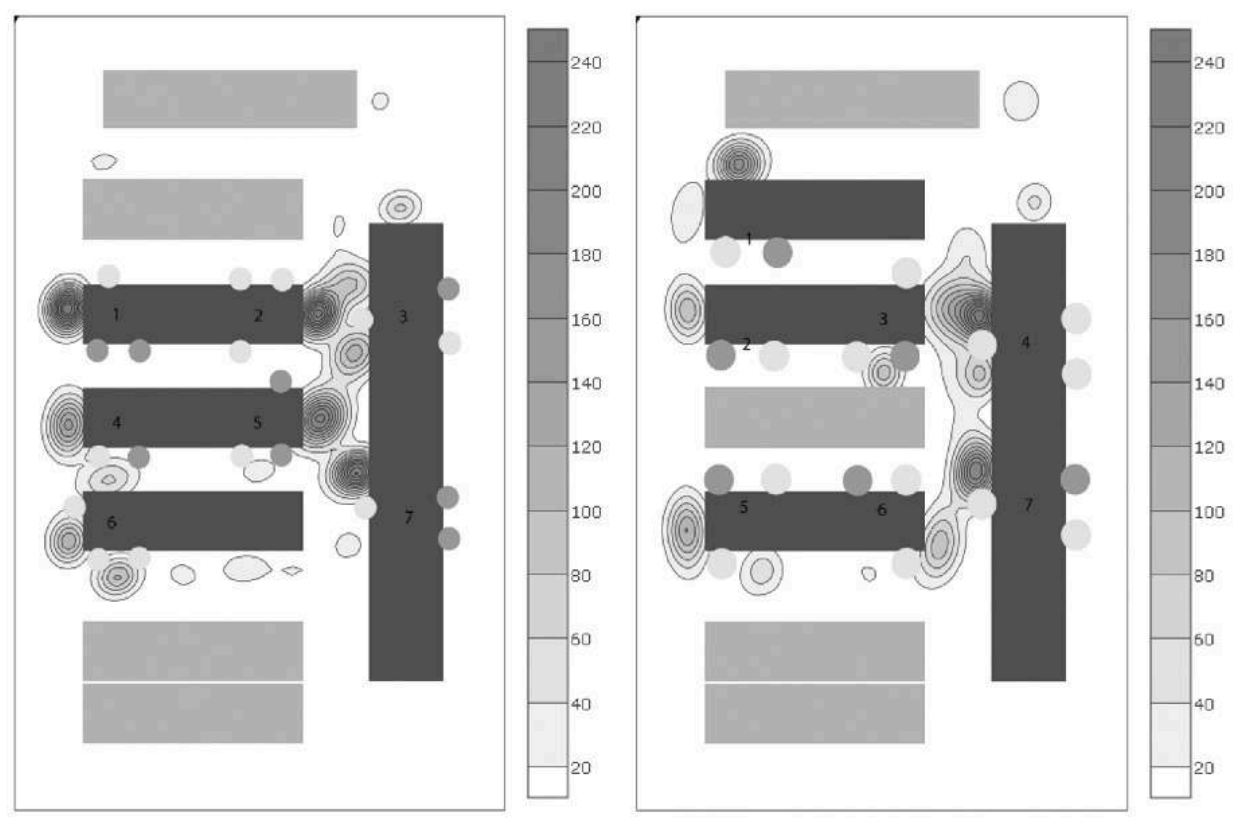

Pour les deux classes, les valeurs de proximité les plus grandes (signifiant que l'enseignante est plus éloignée des groupes considérés) sont celles des groupes proches de la travée de gauche. Les valeurs les plus faibles correspondent aux groupes proches de la travée centrale. La présence de l'enseignante est plus marquée pour les quatre groupes proches de la travée centrale, elle se trouve en moyenne à $3,7 \mathrm{~m}$ des groupes d'élèves donnant sur la travée centrale, et à $5,4 \mathrm{~m}$ de ceux qui donnent sur la travée de gauche. D'après le test de Student $(t=5.22, d f=11.95, \mathrm{p}<0.001)$, la différence est statistiquement significative.

Nous faisons l'hypothèse que la présence de la table latérale concentre les déplacements dans la travée centrale et crée une dissymétrie dans les interactions effectives avec les groupes d'élèves. Cette observation laisse supposer que les modifications dans le placement de l'enseignante qui résultent des contraintes de 
passabilité ont des conséquences différentielles sur ses interactions avec les groupes d'élèves.

\section{Discussion et conclusion}

\section{Les configurations îlots et bus favorisent la proximité dans les interactions avec les élèves} manière à ce qu'ils soient face-à-face. Un entretien informel avec cette enseignante nous a appris qu'elle a déjà dans son parcours professionnel travaillé en îlots, qu'elle est intéressée par les problématiques du travail en groupes et qu'elle apprécie cette configuration. Il serait intéressant de compléter de futures études par des entretiens et questionnaires afin de cerner les avantages et inconvénients perçus dans chaque configuration et appréhender les connaissances professionnelles qui sous-tendent les décisions de placements, afin de structurer des parcours de formation initiale permettant aux néo-titulaires d'appréhender les possibilités et contraintes spatiales, ainsi que suggéré par Weinstein (1992).

\section{Les contraintes d'accessibilité modifient la proximité réelle de l'enseignant}

Les résultats montrent que des configurations qui présentent des obstacles devant être contournés altèrent la proximité réelle de l'enseignant. En particulier, la configuration en peigne est caractérisée par une forte valeur d'accessibilité potentielle (difficulté d'accès) et une forte valeur de distance de proximité (éloignement de l'enseignant par rapport aux groupes d'élèves). L'absence de travée centrale dans cette configuration oblige l'enseignant à faire tout le tour de la salle pour se déplacer entre les groupes, ce qui rallonge ses déplacements dans la classe. L'existence de cette configuration conduit à s'interroger sur les éléments qui ont conduit à la conception de cet espace. Quel degré de contrôle à l'enseignant sur ce type de choix ? La consultation des professionnels lors de la conception des espaces scolaires apparaît comme un élément fréquemment recommandé (Darmody, Smyth, \& Doherty, 2010 ; Lippman, 2010).

Dans la salle en configuration hybride, les résultats suggèrent que la présence de la table latérale concentre les déplacements de l'enseignant dans une partie de la classe, et crée des inégalités spatiales dans la proximité réelle. On peut s'interroger sur les éléments qui ont guidé l'enseignante pour le choix du placement des élèves, sur la part de choix qui reste ou non du ressort des élèves, sur les caractéristiques des élèves et leurs motivations, sur les connaissances professionnelles qui ont guidé l'enseignante dans l'établissement de ce plan de classe. Il serait intéressant de compléter ce type d'étude par des entretiens, afin d'accéder à ces éléments et de les confronter à des 
études concernant le placement des élèves et la réussite scolaire comme celles de Tagliacollo, Volpato, et Pereira (2010) ou de Rißler, Bossen et Blasse (2014)

Nous remarquons aussi que dans toutes les configurations, la proximité réelle est plus faible que l'accessibilité potentielle (Figure 6). L'enseignant compense dans ses déplacements les contraintes d'accessibilité. La différence la plus marquée correspond à la configuration Peigne, on peut supposer que l'énergie dépensée pour cette compensation est d'autant plus forte et la fatigue qui en résulte est vraisemblablement plus marquée.

\section{Les contraintes de passabilité modifient les placements de l'enseignant dans ses interactions avec les élèves}

Les résultats montrent qu'un espace entre rangs trop faible modifie les placements de l'enseignant. L'enseignant se place latéralement par rapport au groupe, au lieu de se placer en face à face.

Sur le plan pratique, pour ne pas gêner ou empêcher des déplacements, il semble important de respecter une largeur minimale entre rangs de l'ordre de $90 \mathrm{~cm}$ à $1 \mathrm{~m}$. Ce constat est cohérent avec certaines recommandations émanant des corps d'inspection comme les préconisations de Bellamy \& Grandpré (2018) pour les sciences expérimentales.

\section{Recommandations pour la conception d'espaces de classe facilitant les déplacements et interactions et perspectives de recherche}

60 Nos résultats montrent que la configuration en îlots étudiée présente la meilleure accessibilité dans le contexte du travail en petits groupes pour l'enseignement des sciences fondé sur l'investigation.

61 Il serait intéressant de comparer différentes configurations en îlots du point de vue de leur accessibilité. En particulier, on peut se demander si un îlot central est pertinent, quel nombre d'élèves et quelles dimensions des tables sont les plus favorables aux déplacements de l'enseignant et aux interactions?

Il serait intéressant aussi de coupler les mesures d'accessibilité avec des questionnaires et des entretiens avec les utilisateurs, enseignants et élèves, afin d'examiner les liens possibles entre accessibilité et bien-être.

Comparer les préoccupations des enseignants au moment de leur préparation de séance (Jeannin, 2006) et la pratique enseignante (Robert 1999, Rogalski 1999) en classe avec les déplacements peut permettre de rendre compte de l'impact de la configuration spatiale sur les indices relevés par l'enseignant et qui le conduisent à réguler son action et situation (Grangeat, 2010).

Nous recommandons également d'envisager des solutions permettant de transformer une configuration bus en une configuration îlots, en prévoyant par exemple d'équiper pour moitié la salle de paillasses sèches mobiles facilement déplaçables, équipées par exemple de roulettes bloquantes, comme le proposent Bellamy \& Grandpré (2018). 


\section{Quelles conditions pour diminuer les stresseurs environnementaux ? du point de vue de l'enseignant, plus particulièrement de ses possibilités de déplacement, facteur de mieux être professionnel}

Nos résultats montrent que des valeurs élevées d'accessibilité potentielle sont synonymes d'allongement des déplacements et d'éloignement de l'enseignant. Ils montrent aussi que des contraintes de passabilité liées à la largeur de travées constituent une gêne voire un empêchement de certains placements et déplacements, et modifient la répartition des interactions dans la classe. Un espace inapproprié est de ce fait un stresseur environnemental au sens de H. Seyle qui peut accroître la fatigue des enseignants et générer des inégalités de traitement des élèves. Le respect de largeurs minimales entre rangs et de travées de $90 \mathrm{~cm}$, et les calculs préalables de l'accessibilité potentielle et du triplet de mobilité sont les critères que nous proposons pour estimer en première approche la qualité des espaces de classe, à rénover, réhabiliter ou construire.

\section{BIBLIOGRAPHIE}

Altet, M. (1994). Note de synthèse, Comment interagissent enseignant et élèves en classe ? Revue française de pédagogie, 107(1), 123-139.

Bellamy, J-M \& Grandpré, C. (2018). Préconisations pour l'aménagement des salles de sciences en collège. Inspection de Sciences et Vie de la Terre de l'académie de Rouen. En ligne : http://svt.spip.acrouen.fr/sites/svt.spip.ac-rouen.fr/IMG/pdf/

20170129_preconisations_salles_de_sciences_colleges_v3.pdf

Brooks, D. C. (2011). Space matters: The impact of formal learning environments on student learning. British Journal of Educational Technology, 42(5), 719-726.

Brooks, D. C. (2012). Space and consequences: The impact of different formal learning spaces on instructor and student behavior. Journal of Learning Spaces, 1(2).

Bucheton, D., \& Soulé, Y. (2009). Les gestes professionnels et le jeu des postures de l'enseignant dans la classe : un multi-agenda de préoccupations enchâssées. Éducation et didactique, 3(3), 29-48.

Clot, Y. (2014). Travail et pouvoir d'agir. Paris : Presses universitaires de France.

Clot, Y. (2015). Le travail à cœur : pour en finir avec les risques psychosociaux. Paris : La découverte.

Clot, Y., Faïta, D., Fernandez, G., \& Scheller, L. (2001). Entretiens en autoconfrontation croisée : une méthode en clinique de l'activité. Education permanente, 146(1), 17-25.

Darmody, M., Smyth, E., \&Doherty, C. (2010). Designing primary schools for the future. Economic and Social Research Institute (ESRI) Research Series.

Dumolard, P. (1999). Accessibilité et diffusion spatiale. L'espace Géographique, 205-214. 
Fischer, G. N., et Fousse, C. (2002). Espaces de travail et communication - Une lecture psychosociale. Communication et organisation, (21).

Fischer, G. (2011). Psychologie sociale de l'environnement. Paris : Dunod

Forest, D. (2006). Analyse proxémique d'interactions didactiques. Carrefours de l'éducation, (1), 73-94.

Guérin, F., Laville, A., Daniellou, F., Duraffourg, J., \& Kerguelen, A. (1991 ; 2007). Comprendre le travail pour le transformer : la pratique de l'ergonomie. Lyon, ANACT.

Grangeat, M. (2010). Les régulations métacognitives dans l'activité enseignante : rôle et modes de développement. Revue des sciences de l'éducation, 36(1), 233-253.

Hall, E.T. (1966). La dimension cachée.

Howells, L. T., et Becker, S. W. (1962). Seating arrangement and leadership emergence. The Journal of Abnormal and Social Psychology, 64(2), 148.

Issaadi, S., et Jaillet, A. (2017). Proxémie d'apprentissage. Éducation et socialisation. Les Cahiers du CERFEE, (43).

Jeannin L. (2006). Appropriation par un enseignant de physique d'une nouvelle séquence d'enseignement : cas de la mécanique en seconde, Thèse de doctorat

Jeannin, L. (2017). La mobilité, clé de nouvelles pratiques ? Éducation et socialisation. Les Cahiers du CERFEE, (43).

Jourdan, I. (2014). Posture, corps et voix de l'enseignant débutant : Une démarche clinique de formation. Recherches \& éducations, (12), 105-116.

Karwowski, W. (2005). Ergonomics and human factors: the paradigms for science, engineering, design, technology and management of human-compatible systems. Ergonomics, 48(5), 436-463.

Laugaa, D. (2004). Stress et burnout des enseignants en école élémentaire. Une approche transactionnelle (Doctoral dissertation, Thèse de doctorat en Psychologie, Université de Bordeaux 2).

Le Cœur, M. (2011). La chaire et les gradins : De la salle de classe à la salle de cours dans les lycées au XIX e siècle. Histoire de l'éducation, 85-109.

Lécuyer, R. (1975). Psychosociologie de l'espace. I. Disposition spatiale et communication en groupe. L'année psychologique, 75(2), 549-573.

Leplat, J. (1997). Regards sur l'activité en situation de travail : contribution à la psychologie ergonomique. Paris : Presses universitaires de France.

Lermigeaux-Sarrade, I. (2018). Rôle de la configuration de l'espace de travail dans les activités effectives et empêchées des enseignants. (Doctoral dissertation, Université Grenoble Alpes).

Lippman, P. C. (2010). Can the Physical Environment Have an Impact on the Learning Environment?(No. 2010/13). OECD Publishing.

Morgado, N. (2014). L'influence des contraintes physiques et sociales des actions sur la perception visuelle de l'espace (Doctoral dissertation, Université de Grenoble).

Moulin, J. F. (2004). Le discours silencieux du corps enseignant. Carrefours de l'éducation, (1), 142-159.

Parzen, E. (1962). On estimation of a probability density function and mode. The annals of mathematical statistics, 33(3), 1065-1076. 
Perkins, K. K., \& Wieman, C. E. (2005). The surprising impact of seat location on student performance. The Physics Teacher, 43(1), 30-33.

Rascle, N., \& Bergugnat, L. (2016). Qualité de vie des enseignants en relation avec celle des élèves : revue de question, recommandations. Contribution dans le cadre du rapport du Cnesco sur la qualité de vie à l'école.

Ria, L. (2008). Ergonomie du travail enseignant.

Rißler, G., Bossen, A., \& Blasse, N. (2014). School as space: spatial alterations, teaching, social motives, and practices. Studia paedagogica, 19(4), 145.

Robert, A. (1999). Recherches didactiques sur la formation professionnelle des enseignants de mathématiques du second degré et leurs pratiques en classe, Didaskalia, 15, 123-157

Rogalski, J. (1999). Approche de psychologie ergonomique de l'activité de l'enseignant. Communication au Stage national COPIRELEM. Limoges

Selye, H. (1946). The general adaptation syndrome and the diseases of adaptation. The journal of clinical endocrinology, 6(2), 117-230.

Sommer, R. (1961). Leadership and group geography. Sociometry, 24(1), 99-110.

Sommer, R. (1965). Further studies of small group ecology. Sociometry, 337-348.

Sommer, R. (1967). Sociofugal space. American Journal of Sociology, 72(6), 654-660.

Stewart, T. M. (2015). The relationship between demands and resources and teacher burnout: A fifteen-year meta-analysis.

Tagliacollo, V. A., Volpato, G. L., \& Pereira Júnior, A. (2010). Association of student position in classroom and school performance. Educational Research, 198-201.

Vickerman, R. W. (1974). Accessibility, attraction, and potential: a review of some concepts and their use in determining mobility. Environment and Planning A, 6(6), 675-691.

Wannarka, R., \& Ruhl, K. (2008). Seating arrangements that promote positive academic and behavioural outcomes: A review of empirical research. Support for learning, 23(2), 89-93.

Weinstein, C. S. (1992). Designing the Instructional Environment: Focus on Seating.

\section{RÉSUMÉS}

Cet article questionne le bien-être au travail de l'enseignant en relation avec sa liberté de déplacement dans l'espace classe et son impact sur les interactions avec les élèves. Le contexte d'étude est le travail en groupes, en démarche d'investigation en sciences, au collège. Le stress professionnel de l'enseignant a fait l'objet de nombreuses études, et les recherches en analyse de l'activité et ergonomie du travail reconnaissent l'activité empêchée comme facteur de stress (Clot, 2015 ; Karwowski, 2005 ; Stewart, 2015). Nous nous intéressons ici en particulier aux déplacements empêchés de l'enseignant, révélateurs de compromis entre ses buts, sa mobilité, les relations de proxémie, l'espace social et les interactions de classe (Brooks, 2011 ; Forest, 2006 ; Hall, 1966, Jeannin, 2017 ; Weinstein, 1992). Avec une méthodologie spécifique (LermigeauxSarrade, 2018), nous comparons les déplacements réels des enseignants dans quatre configurations de classe. Nous montrons que les configurations Îlots et Bus, favorisent la proximité dans les interactions enseignant-élèves, et que les contraintes d'accessibilité qui rallongent tout ou partie des déplacements créent des inégalités dans la proximité réelle 
enseignant-élèves, et empêchent des placements favorables aux interactions. Nous formulons des recommandations pour la conception d'espaces de classe « sociopètes » facilitant les interactions.

This paper questions the teacher's well-being at work in relation to his freedom of movement in the classroom and its impact on interactions with students. The context of the study is groupworking, during inquiry based sciences teaching. The professional stress of the teacher has been the subject of many studies, and research in activity analysis and ergonomics of work. recognize activity as a stressor (Clot 2015, Karwowski 2005, Stewart 2015). Specifically, we focus on the teacher's impeded movements, revealing the trade-off between the teacher's goals, mobility, the proxemic relations, the social space, and the interactions in the classroom (Brooks, 2011, Forest, 2010, Hall, 1966, Jeannin, 2017, Weinstein, 1992). Using a specific methodology (LermigeauxSarrade, 2018), we compared the actual movements of teachers in four classroom configurations. Results showed that the Island and Bus configurations promote proximity in teacher-student interactions, and that the accessibility constrains that lengthen all or parts of the trips create inequities in the actual teacher-student proximity and prevent favourable placements. We make recommendations for the design of "sociopetal" classroom spaces facilitating interactions.

INDEX

Keywords : stress at work, teacher activity, potential accessibility, actual proximity

Mots-clés : stress au travail, activité enseignant, accessibilité potentielle, proximité réelle

\section{AUTEURS}

\section{ISABELLE LERMIGEAUX-SARRADE}

Université Grenoble-Alpes, laboratoire LaRAC (EA 602)

\section{LAURENT JEANNIN}

Université de Cergy-Pontoise, MCF, laboratoire BONHEURS (EA 7517), titulaire de la chaire de recherche Transition2 : des espaces en transition à la transition des espaces 